\title{
Features of creatine-kinase in COVID-19 patients with different ages, clinical types and outcomes: A
}

\section{cohort study}

Authors: Shanshan Wan ${ }^{\mathrm{a}, 1}$, Gaojing Qu ${ }^{\mathrm{b}}$, Hui Yu ${ }^{\mathrm{b}}$, Haoming Zhu ${ }^{\mathrm{b}}$, Guoxin Huang ${ }^{\mathrm{b}}$, Lei Chen ${ }^{\mathrm{b}}$, Meiling Zhang ${ }^{\mathrm{b}}$, Jiangtao Liu ${ }^{\mathrm{c}, *}$, Bin Pei ${ }^{\mathrm{b}, *}$

\author{
Affiliations: \\ a Postgraduate Training Basement of Jinzhou Medical University, Xiangyang No.1 People's Hospital, Hubei University of Medicine, Xiangyang \\ 441000, China \\ b Center of Evidence-based Medicine, Xiangyang No.1 People's Hospital, Hubei University of Medicine, Xiangyang 441000, China \\ c Department of Orthopedics, Xiangyang No.1 People's Hospital, Hubei University of Medicine, Xiangyang 441000, China \\ ${ }^{*}$ Corresponding author
}

\section{Correspondence:}

Professor Jiangtao Liu, Department of Orthopedics, Xiangyang No.1 People's Hospital, Hubei University of Medicine, Xiangyang 441000, Hubei Province, China. E-mail: 1jt73@sohu.com; Phone: +86-13972236555.

Professor Bin Pei, Center of Evidence-based Medicine, Xiangyang No.1 People's Hospital, Hubei University of Medicine, Xiangyang 441000, Hubei Province, China. E-mail: binpei@hbmu.edu.cn; Phone: +86-18995678520. 


\section{Author details:}

Shanshan Wan ${ }^{\mathrm{a}, 1}$, wss29530503@163.com.

Gaojing Qu ${ }^{\mathrm{b}}$, xzyxqgj@163.com.

Hui Yu ${ }^{\mathrm{b}}$, yuhui06127@163.com

Haoming Zhu ${ }^{\text {b }}$, zhuhaoming_hbmu@,163.com

Guoxin Huang ${ }^{\mathrm{b}}$, xzyxhgx@163.com

Lei Chen ${ }^{\mathrm{b}}$, chenleimedical@163.com

Meiling Zhang ${ }^{\mathrm{b}}$,506989038@qq.com

Jiangtao Liu ${ }^{\mathrm{c}, *}, 1 \mathrm{jt73} @$ sohu.com

Bin Peib, ${ }^{\text {b* }}$ binpei@hbmu.edu.cn 


\begin{abstract}
Objectives: To study the features of creatine-kinase (CK) in COVID-19 patients with different ages, clinical types and outcomes and quantify the relationship between CK value and clinical type.

Methods: All laboratory confirmed COVID-19 patients hospitalized in Xiangyang No.1 People's Hospital were included. Patients' general information, clinical type, all CK values and outcome were collected.

Results: The peak median value of CK in cases aged $\geq 71$ years old (appeared at T2) was higher than that in cases aged $\leq 70$ years old. There was statistical difference between the two groups $(P=0.001)$. Similarly, the peak in critical cases (appeared at T2) was higher than moderate and severe types, and significant difference were existed among moderate, severe, and critical types $(P=0.000)$. Moreover, the peak value in death group (appeared at T2) was higher than those in survival group. Significant difference was also found between them $(P=0.000)$. According to the optimal scale regression model, the $\mathrm{CK}$ value $(P=0.000)$ and age $(P=0.000)$ were associated with the clinical type.

Conclusions: Difference of the CK in different ages, clinical types, and outcomes were significant. The results of the optimal scale regression model are helpful to judge the clinical type of COVID-19 patients.
\end{abstract}

KEY WORDS: COVID-19, creatine-kinase, feature, cohort study.

\title{
1. Introduction
}


Coronavirus disease-19(COVID-19), a virus induced pneumonia, has spread rapidly around the world[1-3]. Creatine-kinase (CK) is an important kinase directly related to intracellular energy transport, muscle contraction and ATP regeneration. It can reversibly catalyze the following reaction: ATP + creatine $\rightarrow$ ADP + phosphocreatine[4, 5], provide energy for muscle contraction and transport system[6]. CK was mainly found in the myocardium, skeletal muscle and brain, while little distributed in lung, gastrointestinal tract and thyroid. Sufficient CK can be released and terminally enhance the activity of these tissues under the pathological state[7]. Chen et al. indicated that CK abnormally increased in $13(13.0 \%)$ out of 99 COVID-19 cases[8].

Two Meta analyses showed that the increase of CK value was significantly correlated to the severity of COVID-19 patients, and there was significant difference of $\mathrm{CK}$ value between severe and non-severe COVID-19 patients $(P<0.01)[9,10]$. Zhou, Zheng et al. conducted a retrospective analysis of COVID-19 patients and indicated that CK value was significant higher in severe patients than those in mild patients. It was reported that the increase of CK could be served as a predictor of the severity of the disease[11, 12]. Chen Tao et al. described the clinical characteristics of 113 dead COVID-19 patients. According to their research, the median value of CK in the death group (113.0 U/L) was higher than that in the rehabilitation group (161.0 U/L)[13], but they did not conduct a statistical analysis of the differences. A univariate analysis showed that $\mathrm{CK}>185 \mathrm{U} / \mathrm{L}$ was associated with the death of COVID-19 patients[14].

At present, there is a lack of systematic research on the changes of CK value in COVID-19 patients with different ages, clinical types and outcomes, and the discrimination of clinical type with CK value still needs in-depth analysis. Therefore, we collected all the confirmed COVID-19 patients in Xiangyang No.1 People's hospital, and analyzed the CK value since admission. 


\section{Methods}

\subsection{Study design and patients}

This study was a bidirectional observational cohort study and the cohort was established on Feb 9, 2020. All suspected and confirmed cases of COVID-19 admitted to the Xiangyang No.1 People's Hospital Affiliated Hospital of Hubei University of Medicine according to the Diagnosis and Treatment Protocol for Novel Coronavirus Pneumonia (1st-7th editions) were included in this study[15]. The retrospective data were traced back to Jan 22, 2020 and the follow-up was carried out until Mar 28, 2020. Patients were classified into mild, moderate, severe, and critical type according to diagnosis and treatment protocol[15]. The classification results were cross-checked by two experts, and a third specialist involved if the inconsistency existed. The study was approved by the ethics review board of Xiangyang No.1 People's Hospital (No. 2020GCP012) and registered at the Chinese Clinical Trial Registry as ChiCTR2000031088. Informed consent from patients has been exempted since this study does not involve patients' personal privacy neither incur greater than the minimal risk.

\subsection{Data collection}

Data were extracted from the hospital information system by two groups and then cross-checked. Sex, age, CK value, disease onset date, outcome were collected.

\subsection{Outcome measures}

In this cohort, symptom onset was regarded as disease onset and the corresponding date was set as the day 1 to record data from symptom 
onset. Since the time from symptom onset to discharge was $26.87 \pm 9.19$ days, we record the data from day 1 to day 30 . The difference during day 5 to day 15 was most obvious, so we conduct statistical analysis on this period. We draw the CK median value/(5 days) trend chart with 5 days as the time unit. T1,T2...Tn was successively used to record per 5 days time unit, for example, T1 means the first 5 days after the onset of the disease. Mild type were excluded when we conducted the comparative and statistical analysis, because it only contained 4 cases.

\subsection{Statistical analysis}

All statistical analyses were performed through SPSS 20.0. Binary data were described by frequency and percentage. The normality of continuous data was checked. Mean and standard deviation were used to describe variables with normal distribution; otherwise, median (interquartile, IQR) was applied. Categorical data were described as frequency (\%); the chi-square test was applied to assess significance between groups. Optimal scale regression model is used for regression analysis. All graphs were processed through GraphPad Prism 8.0 and Photoshop CC 14.2 software.

\section{Results}

This study included all of the suspected and laboratory-confirmed 542 patients till Feb 28, 2020. Among the 542 cases, the nucleic acid tests in 142 cases were positive. Excluding 2 infants, and 9 cases that transferred from other hospital whose data cannot be traced, 131 cases were included in the further study finally.

\subsection{General information}


Among the included 131 cases, there were 63 males and 68 females, and the average age was $50.1 \pm 17.1$ years old. The average age was 48.2 \pm 16.2 and 73.1 \pm 9.5 years old in survival group and death group, respectively. The average time from onset to admission, from onset to discharge, from onset to death, and length of hospitalization were $4.5 \pm 3.1,26.9 \pm 9.2,18.4 \pm 9.8$ and $22.4 \pm 8.7$ days respectively. 121 cases were discharged from the hospital while 10 cases died.

\subsection{CK test results}

The 131 cases underwent 37 laboratory indicators contained 24052 tests in outpatient and hospitalization. This cohort included all of the CK results, totally 504 times of test, account for $2.1 \%$ of the all results of indicators. The normal range of CK value is $50.0-310.0 \mathrm{U} / \mathrm{L}$. The change trend of CK median value showed a downward trend since the symptom onset (Supplement Figure A).

\subsection{The features of $C K$ in different ages}

Cases were divided into 5 groups including $\leq 40,41-50,51-60,61-70$, and $\geq 71$ years old group, while only the $\geq 71$ years old group hold a obviously different distribution from other groups (Supplement Figure B). Moreover, significant difference of the CK median value was observed between the divided groups $(P=0.000)$, while no statistical difference was found after excluding the $\geq 71$ years old group $(P=0.083)$ (Supplement Table). The age was correlated with CK value $(P=0.000)$ with the coefficient as 0.235 in the five age groups ( $\leq 40,41-50,51-60$, $61-70, \geq 71$ years old group); while no correlation was found between age and CK value after excluding the $\geq 71$ years old group $(P=0.344)$.

Based on the above results, we divided the cases into $\leq 70$, and $\geq 71$ years old group. 
In $\leq 70$ years old group and $\geq 71$ years old group, the CK median value was $50.9 \mathrm{U} / \mathrm{L}$ and $59.7 \mathrm{U} / \mathrm{L}$, respectively, and the CK value abnormal percentage was $3.9 \%$ and $13.5 \%$, respectively. The changes indicated that the CK median value in $\leq 70$ years old group decreased during T1-T6, and the peak value (76.9 U/L) distributed at T1; in $\geq 71$ years old group, the CK median value increased during T1-T2 and decreased after T2, and the peak value (205.7 U/L) distributed at T2. The CK median value all within normal range among the groups (Table 1, Fig. 1A).

Statistical analysis of CK value from day 5 to day 15 showed statistical difference between the two groups $(P=0.001)$, and the CK median value was higher in $\geq 71$ years old group than those in $\leq 70$ years old group.

\subsection{The features of $C K$ in different clinical types}

In mild group, moderate group, severe group and critical group, the $\mathrm{CK}$ median value were 56.4 U/L, 46.2 U/L, 51.3 U/L and 79.50 U/L, respectively, and the abnormal percentage of CK value was $0.0 \%, 1.1 \%, 2.1 \%$ and $19.8 \%$, respectively. The changes indicated that the CK median value of the moderate group and severe group decreased during T1-T6, and the peak value (67.4 U/L and 168.9 U/L, respectively) distributed at T1; in the critical group, the CK median value increased during T1-T2 and decreased after T2, and the peak value (283.0 U/L) distributed at T2. The CK median value all within normal range among the groups (Table 2, Fig. 1B).

Statistical analysis of CK value from day 5 to day 15 showed statistical difference among the three clinical type groups $(P=0.000)$. The $\mathrm{CK}$ median value in severe type was significantly higher than those in moderate type $(P=0.006)$. The CK median value in critical type was significantly higher than those in moderate type $(P=0.000)$ and severe type $(P=0.004)$. 
The maximum CK in the first 15 days after symptom onset was collected to build the categorical regression based on optimal scale. For the reason that the mild type only contained 4 cases, we excluded this type. The model output showed that age $(P=0.000)$ and $\mathrm{CK}(P=0.000)$ had significant correlation while sex $(P=0.253)$ did not have significant correlation with clinical type. The quantification of CK categories showed $\mathrm{CK} \leq 310.33 \mathrm{U} / \mathrm{L}$ was related to moderate type. $\mathrm{CK}$ ranged $360.37-883.44 \mathrm{U} / \mathrm{L}$ was related to severe type, and $\mathrm{CK}>883.44 \mathrm{U} / \mathrm{L}$ was related to critical type. The quantification of age showed $\leq 61$ years old was related to moderate type, 62-70 years old was related to the severe type, and 71-90 years old was related to critical type (Table 4). The model expression was Q_level=0.311*Q_CK+0.525*Q_age+0.059*Q_sex $\left(\mathrm{Q} \_l\right.$ level, Q_CK, Q_age and Q_sex represent the scale quantization score of clinical type, CK value, age and sex, respectively). The comparison between severity quantification of different clinical type output by this model and the actual severity quantification of the clinical type classified by the respiratory physicians was shown in Fig.1D. The accuracy rate of this output on three types (moderate, severe, critical) was $73.6 \%$. The value of the model below 1 is divided into moderate type and severe type, and over 1 is divided into critical type because only the critical type hold a obviously different distribution from other clinical types (Fig. 1B). The accuracy of distinguishing the two types is $89.6 \%$.

\subsection{The features of $C K$ in different outcomes}

In survival group and death group, the $\mathrm{CK}$ median value was $48.9 \mathrm{U} / \mathrm{L}$ and $138.7 \mathrm{U} / \mathrm{L}$, respectively, and the $\mathrm{CK}$ value abnormal percentage was $2.7 \%$ and $32.7 \%$, respectively. The changes indicated that the CK median value in survival group decreased during T1-T6, and the peak value (79.4 U/L) distributed at T1; while in death group, the CK median value increased during T1-T2 and decreased after T2, and the peak value (403.4 U/L) distributed at T2, the abnormal time interval was T2 (Table 3, Fig. 1C). 
Statistical analysis of CK value from day 5 to day 15 showed statistical difference between the two outcome groups $(P=0.000)$, and the CK value in death group was higher than those in survival group. According to the spearman correlation analysis, the correlation was found between outcome and $\mathrm{CK}$ value $(P=0.000)$ with the coefficient as 0.341 in the two outcome groups.

\section{Discussion}

The CK value in cases aged $\geq 71$ years old group increased during day 5 to day 15 , which was significantly different from other age groups. The correlation analysis suggested a correlation between the $\geq 71$ years old group and the CK value, indicating that among the five age groups, cases aged $\geq 71$ years old had the most severe cell injury and induced the increased CK value. CK is mainly distributed in high energy metabolic organs such as heart and brain, indicating that important organs are more likely to be damaged in cases aged $\geq 71$ years old. This is consistent with the result of our regression analysis that 71-90 years old was related to critical type (Table 4). It is suggested that we should pay close attention to the changes of $\mathrm{CK}$ value in cases aged $\geq 71$ years old, and this is consistent with the multivariate regression analysis conducted by Zhou et al, which showed that the death of COVID-19 patients usually associated with the advanced age[14]. But the reason that the important hypermetabolic organs in cases aged $\geq 71$ years old are prone to serious damaged worth further study.

There were significant differences among different clinical types, and the more serious the type , the higher the peak value of CK, and the greater the proportion of CK over ULN. At T2, significant differences were found in the CK value among the three clinical types, suggesting that T2 may be the period for judging the clinical type of COVID-19 patients, CK during T2 period should be closely monitored, and CK > 197.0 U/L (upper quartile for severe type) may develop into critical type. Therefore, we recommend that clinicians should closely monitor the CK 
value in the early stage of COVID-19 patients for early warning and intervention. In order to quantify the relationship between CK value and clinical type, regression analysis was conducted based on CK, age, and sex. Since the time from onset to severe type and critical type is $8.15 \pm$ 5.29 days and $11.83 \pm 5.19$ days respectively, the maximum values of CK in the first 15 days were selected in this model. The results show that the CK value can be used to distinguish clinical types, which can help us to grasp the opportunity of treatment better and prevent patients from developing into critical type. However, the prediction ability of this model need to be verified by other data.

According to the difference between different outcomes, we found that the death group had higher peak value of CK and the greater proportion of CK exceeding ULN. Statistical analysis showed that there was statistical difference between the two outcome groups. Spearman correlation analysis showed that outcome was correlated with CK value. At T2 and T3, the CK value of the death group was significantly higher than that of survival group ( $P=0.001, P=0.000$ respectively), suggesting that $\mathrm{T} 2$ might be the first period to judge the outcome of the disease. And CK $>137.8 \mathrm{U} / \mathrm{L}$ (upper quartile of survival group) has a risk of death. This is of reference value for us to judge the prognosis, especially in the early stage.

To sum up, patients in different ages, clinical types and outcomes, especially those $\geq 71$ years old, critical and dead patients, the CK median value reached the peak at the day 10, which appeared early and concentrated, and then decreased rapidly. It is suggested that the day 10 may be a good index for early clinical classification, and the most critical time point in the progression of the disease. CK exists in a large number of cells which rapidly regenerated by ATP, such as heart muscle, skeletal muscle and brain tissue, and these tissues are important to maintain the normality of human signs. Once the tissues damaged, the permeability of cell membrane increased, which makes CK continuously released into 
the blood, and induced the increased CK value finally[16]. Therefore, early monitoring of CK value has important reference value for us to judge the severity of the disease and the outcome of the disease.

\section{Limitation}

The current study has several limitations. First, the cases included in our cohort were enrolled from single hospital, the evidence value was limited. Second, due to the limited sample size, the data could not be analyzed and described daily, and some information has been lost. Third, multicenter and large sample studies were needed to verify the optimal scale regression model based on clinical type.

\section{Conclusion}

The results showed that the more serious the clinical type, the higher the peak value of CK. The peak value of CK appeared at day 10 in the $\geq 71$ years old group, critical type and death group, which appeared early and concentrated, indicated that early monitoring of CK value is helpful to better evaluate the disease and is of great significance to the graded treatment of patients, the day 10 after onset (4-5 days after hospitalization) may be the most critical time point. The results of the optimal scale regression model are helpful to judge the clinical type of COVID-19 patients. Spearman correlation analysis showed that outcome was correlated with CK value. From the characteristics of changes in CK value, and our model, we can make up for the deficiency of CK in the early diagnosis, severity assessment and prognosis judgment of COVID-19 patients.

\section{Declaration}




\subsection{Ethics approval and consent to participate}

The study was approved by the ethics review board of Xiangyang No.1 People's Hospital (No. 2020GCP012) and registered at the Chinese

Clinical Trial Registry as ChiCTR2000031088. Informed consent from patients has been exempted since this study does not involve patients' personal privacy neither incur greater than the minimal risk.

\subsection{Funding this research}

This research did not receive any specific grant from funding agencies in the public, commercial, or not-for-profit sectors.

7.3 Conflict of interests

The authors have no competing interest to declare.

\subsection{Acknowledgement}

We want to thank all medical workers for their great contribution and sacrifice during this COVID-19 pandemic. 
Table 1 CK in COVID-19 patients with different age groups

\begin{tabular}{|c|c|c|c|c|c|c|c|c|c|}
\hline \multirow{4}{*}{ Period } & \multicolumn{4}{|c|}{$\leq 70$ Years Old } & \multicolumn{4}{|c|}{$\geq 71$ Years Old } & \multirow{4}{*}{$\begin{array}{l}P \text { value } \\
P=0.001\end{array}$} \\
\hline & \multirow{3}{*}{\multicolumn{2}{|c|}{$\begin{array}{l}\text { Median } \\
(\mathrm{IQR})\end{array}$}} & \multirow{3}{*}{\multicolumn{2}{|c|}{$\begin{array}{c}\text { Abnormal } \\
\text { Percentage } \\
(\%)\end{array}$}} & \multirow{3}{*}{\multicolumn{2}{|c|}{$\begin{array}{l}\text { Median } \\
(\mathrm{IQR})\end{array}$}} & \multirow{3}{*}{\multicolumn{2}{|c|}{$\begin{array}{c}\text { Abnormal } \\
\text { Percentage } \\
(\%)\end{array}$}} & \\
\hline & & & & & & & & & \\
\hline & & & & & & & & & \\
\hline Full Course & 50.9 & $(35.0-83.5)$ & 415 & $3.9 \%$ & 59.7 & $(35.4-203.9)$ & 89 & $13.5 \%$ & - \\
\hline $\mathrm{T} 1$ & 76.9 & $(53.3-118.4)$ & 56 & $5.4 \%$ & 116.9 & $(84.9-312.1)$ & 11 & $27.3 \%$ & - \\
\hline $\mathrm{T} 2$ & 64.6 & $(41.2-124.2)$ & 92 & $5.4 \%$ & 205.7 & $(89.2-710.6)$ & 21 & $33.3 \%$ & 0.000 \\
\hline $\mathrm{T} 3$ & 48.4 & $(34.2-75.2)$ & 73 & $4.1 \%$ & 53.9 & $(38.9-75.5)$ & 17 & $5.9 \%$ & 0.291 \\
\hline $\mathrm{T} 4$ & 45.9 & $(30.1-67.8)$ & 77 & $5.2 \%$ & 51.3 & $(28.2-67.6)$ & 19 & $0.0 \%$ & - \\
\hline $\mathrm{T} 5$ & 43.6 & $(34.4-61.4)$ & 62 & $1.6 \%$ & 38.6 & $(32.3-48.7)$ & 14 & $0.0 \%$ & - \\
\hline T6 & 37.7 & $(26.7-51.6)$ & 55 & $0.0 \%$ & 34.9 & $(24.1-45.7)$ & 7 & $14.3 \%$ & - \\
\hline
\end{tabular}

N: total number of $\mathrm{CK}$ tests in corresponding period. $\quad P$ : P value between the two age groups during day 5 to day 15. 
Table 2 CK in COVID-19 patients with different clinical types

\begin{tabular}{|c|c|c|c|c|c|c|c|c|c|c|c|c|c|}
\hline \multirow[b]{2}{*}{ Period } & \multicolumn{4}{|c|}{ Moderate Type } & \multicolumn{4}{|c|}{ Severe Type } & \multicolumn{4}{|c|}{ Critical Type } & \multirow{2}{*}{$\begin{array}{l}P \text { value } \\
P=0.000\end{array}$} \\
\hline & & $\begin{array}{c}\text { Median } \\
(\mathrm{IQR})\end{array}$ & $\mathrm{N}$ & $\begin{array}{c}\text { Abnormal } \\
\text { Percentage } \\
(\%)\end{array}$ & & $\begin{array}{c}\text { Median } \\
(\mathrm{IQR})\end{array}$ & $\mathrm{N}$ & $\begin{array}{c}\text { Abnormal } \\
\text { Percentage } \\
(\%)\end{array}$ & & $\begin{array}{c}\text { Median } \\
(\mathrm{IQR})\end{array}$ & $\mathrm{N}$ & $\begin{array}{c}\text { Abnormal } \\
\text { Percentage } \\
(\%)\end{array}$ & \\
\hline Full Course & 46.2 & $(34.6-68.0)$ & 273 & $1.1 \%$ & 51.3 & $(32.6-114.3)$ & 95 & $2.1 \%$ & 79.5 & $(38.8-287.6)$ & 116 & $19.8 \%$ & - \\
\hline $\mathrm{T} 1$ & 67.4 & $(50.9-93.4)$ & 41 & $2.4 \%$ & 168.9 & $(113.7-239.9)$ & 12 & $16.7 \%$ & 134.0 & $(92.1-307.0)$ & 10 & $30.0 \%$ & - \\
\hline $\mathrm{T} 2$ & 53.9 & $(39.9-94.8)$ & 64 & $3.1 \%$ & 108.5 & $(59.1-197.0)$ & 21 & $0.0 \%$ & 283.0 & $(88.6-689.5)$ & 22 & $45.5 \%$ & $P 1=0.043, \mathrm{P} 2=0.000, P 3=0.003$ \\
\hline $\mathrm{T} 3$ & 45.6 & $(34.2-55.1)$ & 49 & $0.0 \%$ & 51.0 & $(33.4-76.6)$ & 17 & $0.0 \%$ & 68.3 & $(50.6-276.4)$ & 18 & $22.2 \%$ & $P 2=0.003$ \\
\hline $\mathrm{T} 4$ & 44.6 & $(30.2-57.2)$ & 54 & $0.0 \%$ & 44.0 & $(26.5-68.9)$ & 20 & $0.0 \%$ & 69.5 & $(43.2-278.3)$ & 20 & $20.0 \%$ & - \\
\hline T5 & 40.3 & $(29.3-50.7)$ & 37 & $0.0 \%$ & 37.1 & $(31.7-43.6)$ & 16 & $0.0 \%$ & 80.8 & $(38.7-247.7)$ & 22 & $4.5 \%$ & - \\
\hline $\mathrm{T} 6$ & 37.2 & $(26.9-46.1)$ & 28 & $0.0 \%$ & 39.6 & $(23.6-50.9)$ & 9 & $0.0 \%$ & 37.2 & $(26.9-55.4)$ & 24 & $4.2 \%$ & - \\
\hline
\end{tabular}

$\mathrm{N}$ : total number of $\mathrm{CK}$ tests in corresponding period. $\quad P$ : P value among the three clinical types during day 5 to day $15 . \quad P 1:$ P value between moderate type and severe type in corresponding period. $\quad$ P2: $\mathrm{P}$ value between moderate type and critical type in corresponding period. $\quad$ P3: $\mathrm{P}$ value between severe type and critical type in corresponding period. 
Table 3 CK in COVID-19 patients with different outcomes

\begin{tabular}{|c|c|c|c|c|c|c|c|c|c|}
\hline \multirow{3}{*}{ Period } & \multicolumn{4}{|c|}{ Survival Group } & \multicolumn{4}{|c|}{ Death Group } & \multirow{3}{*}{$\begin{array}{l}P \text { value } \\
P=0.000\end{array}$} \\
\hline & & Median & & Abnormal & & Median & & Abnormal & \\
\hline & & （IQR） & $\mathrm{N}$ & $\begin{array}{c}\text { Percentage } \\
(\%)\end{array}$ & & （IQR） & $\mathrm{N}$ & $\begin{array}{c}\text { Percentage } \\
(\%)\end{array}$ & \\
\hline Full Course & 48.9 & $(34.0-80.4)$ & 453 & $2.6 \%$ & 138.7 & $(59.7-470.0)$ & 49 & $32.7 \%$ & - \\
\hline $\mathrm{T} 1$ & 79.4 & $(53.3-119.3)$ & 60 & $6.7 \%$ & 134.0 & (101.1-299.4) & 6 & $33.3 \%$ & - \\
\hline $\mathrm{T} 2$ & 67.3 & $(41.5-137.8)$ & 100 & $5.0 \%$ & 403.4 & $(79.8-779.4)$ & 12 & $58.3 \%$ & 0.001 \\
\hline $\mathrm{T} 3$ & 46.2 & $(34.2-61.1)$ & 81 & $1.2 \%$ & 203.9 & $(75.5-625.3)$ & 9 & $33.3 \%$ & 0.000 \\
\hline $\mathrm{T} 4$ & 45.0 & $(29.9-64.8)$ & 87 & $1.1 \%$ & 117.5 & $(58.6-685.2)$ & 9 & $33.3 \%$ & - \\
\hline $\mathrm{T} 5$ & 39.1 & $(31.5-55.3)$ & 69 & $1.4 \%$ & 119.4 & $(77.0-285.7)$ & 7 & $0.0 \%$ & - \\
\hline T6 & 35.9 & $(26.2-52.2)$ & 56 & $0.0 \%$ & 39.8 & $(36.2-48.9)$ & 6 & $16.7 \%$ & - \\
\hline
\end{tabular}

$\mathrm{N}$ : total number of CK tests in corresponding period. $\quad P$ : P value between the two outcome groups during day 5 to day 15. 
Table 4 Variable categories and quantification score in optimal scale regression model

\begin{tabular}{|c|c|c|c|}
\hline Variable & Classification & Frequency & Quantification \\
\hline \multirow[t]{3}{*}{ Clinical Type } & Moderate Type & 86 & -0.571 \\
\hline & Severe Type & 21 & 0.382 \\
\hline & Critical Type & 18 & 2.284 \\
\hline \multirow[t]{5}{*}{$\mathrm{CK}$} & $4.05-61.08$ & 43 & -0.705 \\
\hline & $63.01-310.33$ & 70 & -0.043 \\
\hline & $360.37-457.70$ & 6 & 2.229 \\
\hline & $710.57-883.44$ & 3 & 2.229 \\
\hline & $1127.92-4371.26$ & 3 & 4.427 \\
\hline \multirow[t]{2}{*}{ Sex } & Male & 60 & 1.041 \\
\hline & Female & 65 & -0.961 \\
\hline \multirow[t]{7}{*}{ Age } & $15-29$ & 16 & -0.778 \\
\hline & $30-39$ & 20 & -0.536 \\
\hline & $40-47$ & 18 & -0.536 \\
\hline & $48-54$ & 20 & -0.536 \\
\hline & $55-61$ & 15 & -0.536 \\
\hline & $62-70$ & 18 & 0.683 \\
\hline & $71-90$ & 18 & 2.184 \\
\hline
\end{tabular}


A
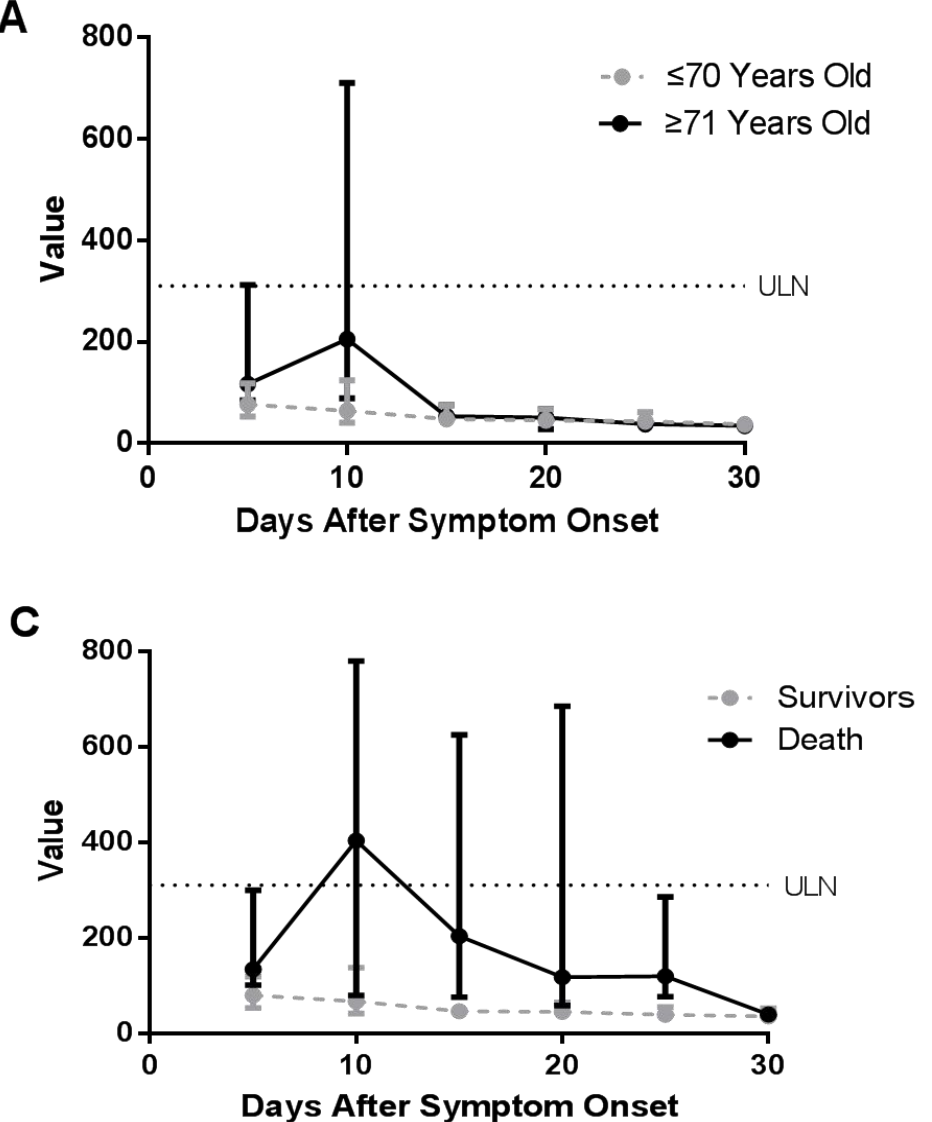

B

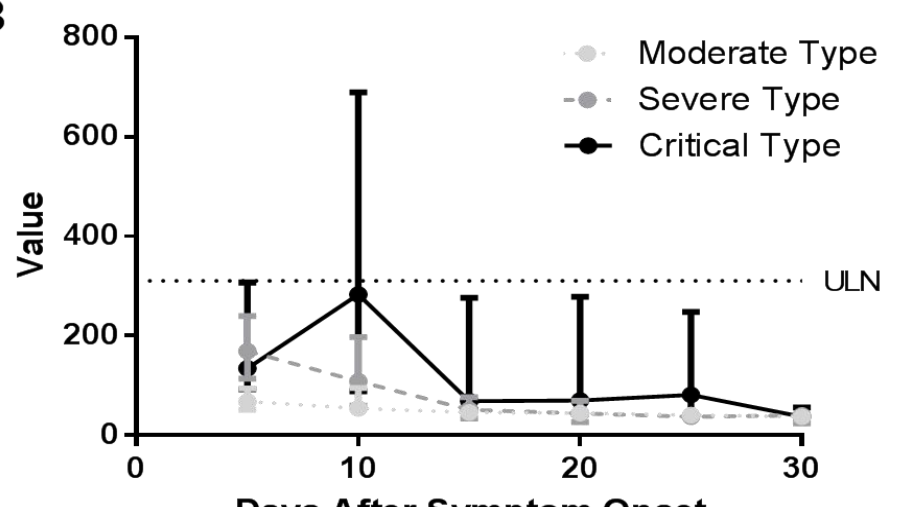

Days After Symptom Onset

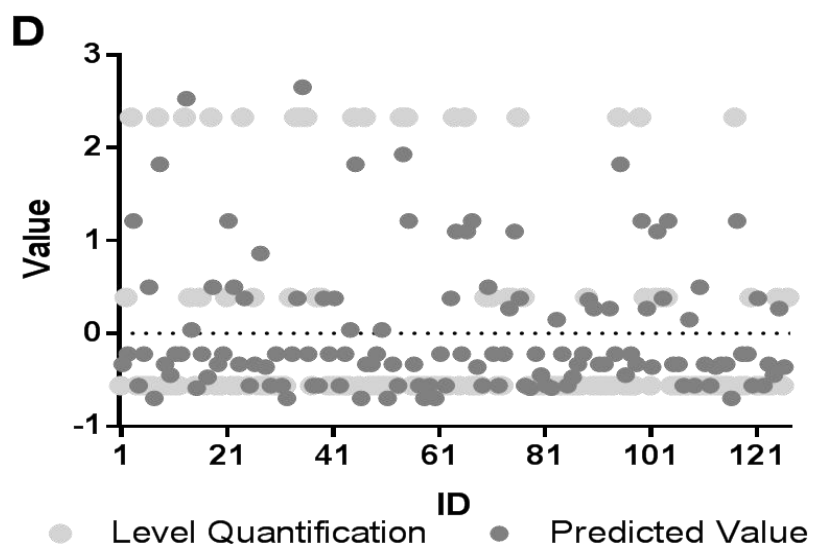

Fig. 1 The change trend of CK median value in different ages (A), clinical types (B), outcomes (C), and comparison of quantitative scores of model output (D). 
Supplement Table CK in COVID-19 patients with five age groups

\begin{tabular}{|c|c|c|c|c|c|c|c|c|}
\hline \multicolumn{2}{|c|}{ Course } & \multirow{2}{*}{$\begin{array}{c}\text { Full } \\
48.4(36.7-73.0)\end{array}$} & \multirow{2}{*}{$\begin{array}{c}\mathrm{T} 1 \\
66.6(51.0-95.9)\end{array}$} & \multirow{2}{*}{$\begin{array}{c}\mathrm{T} 2 \\
54.7(39.4-79.8)\end{array}$} & \multirow{2}{*}{$\begin{array}{c}\mathrm{T} 3 \\
41.6(33.4-53.9)\end{array}$} & \multirow{2}{*}{$\begin{array}{c}\mathrm{T} 4 \\
44.6(30.6-57.5)\end{array}$} & \multirow{2}{*}{$\begin{array}{c}\text { T5 } \\
43.3(36.1-55.3)\end{array}$} & \multirow{2}{*}{$\begin{array}{c}\text { T6 } \\
57.0(44.4-108.0)\end{array}$} \\
\hline$\leq 40$ & Median(IQR) & & & & & & & \\
\hline Years Old & Percentage $(\%)$ & $3.0 \%$ & $9.1 \%$ & $0.0 \%$ & $4.8 \%$ & $0.0 \%$ & $0.0 \%$ & $0.0 \%$ \\
\hline $41-50$ & Median(IQR) & $50.8(34.0-83.2)$ & $96.5(73.8-159.7)$ & $74.3(46.2-197.0)$ & $51.7(41.2-75.9)$ & $46.2(26.1-75.7)$ & $40.2(29.1-56.7)$ & $34.6(25.3-52.2)$ \\
\hline Years Old & Percentage $(\%)$ & $1.0 \%$ & $0.0 \%$ & $4.8 \%$ & $0.0 \%$ & $0.0 \%$ & $0.0 \%$ & $0.0 \%$ \\
\hline $51-60$ & Median(IQR) & $50.0(32.1-76.5)$ & $75.5(48.9-93.4)$ & $86.4(49.5-150.5)$ & $53.1(32.1-82.2)$ & $44.9(29.9-57.9)$ & $39.1(22.1-49.8)$ & $33.5(29.5-40.1)$ \\
\hline Years Old & Percentage( $(\%)$ & $2.7 \%$ & $11.1 \%$ & $5.3 \%$ & $0.0 \%$ & $0.0 \%$ & $0.00 \%$ & $0.0 \%$ \\
\hline $61-70$ & Median(IQR) & $51.6(37.9-103.4)$ & $100.6(72.0-138.8)$ & $68.1(43.2-87.9)$ & $49.1(38.6-58.3)$ & $65.9(31.6-169.2)$ & $58.7(38.3-122.1)$ & $34.8(24.3-43.1)$ \\
\hline Years Old & Percentage $(\%)$ & $9.1 \%$ & $0.0 \%$ & $16.7 \%$ & $9.5 \%$ & $20.0 \%$ & $5.6 \%$ & $0.0 \%$ \\
\hline$\geq 71$ & Median(IQR) & $59.7(35.4-203.9)$ & $116.9(84.9-312.1)$ & $205.7(89.2-710.6)$ & $53.9(38.9-75.5)$ & $51.3(28.2-67.6)$ & $38.6(32.3-48.7)$ & $34.9(24.1-45.7)$ \\
\hline Years Old & Percentage $(\%)$ & $13.5 \%$ & $27.3 \%$ & $33.3 \%$ & $5.9 \%$ & $0.0 \%$ & $0.0 \%$ & $14.3 \%$ \\
\hline \multirow{4}{*}{$\mathrm{P}$ value } & & \multirow{4}{*}{-} & \multirow{4}{*}{-} & $P 1=0.000$ & & \multirow{4}{*}{ - } & \multirow{4}{*}{ - } & \multirow{4}{*}{ - } \\
\hline & $P=0.000$ & & & $P 2=0.011$ & & & & \\
\hline & \multirow[t]{2}{*}{$P^{*}=0.083$} & & & $P 3=0.013$ & & & & \\
\hline & & & & $P 4=0.005$ & & & & \\
\hline
\end{tabular}

Percentage (\%): abnormal rate in corresponding period. $\quad P: \mathrm{P}$ value among the $\leq 40,41-50,51-60,61-70$ and $\geq 71$ years old groups during day 5 to day $15 . \quad P *$ : $\mathrm{P}$ value among the $\leq 40,41-50,51-60$ and $61-70$ years old groups during day 5 to day 15 . $P 1$ : P value between $\leq 40$ and $\geq 71$ years old group in corresponding period. $P 2$ : $\mathrm{P}$ value between $41-50$ and $\geq 71$ years old group in corresponding period. $\quad P 3$ : $\mathrm{P}$ value between $51-60$ and $\geq 71$ years old group in corresponding period. $\quad P 4$ : $\mathrm{P}$ value between $61-70$ and $\geq 71$ years old group in corresponding period. 

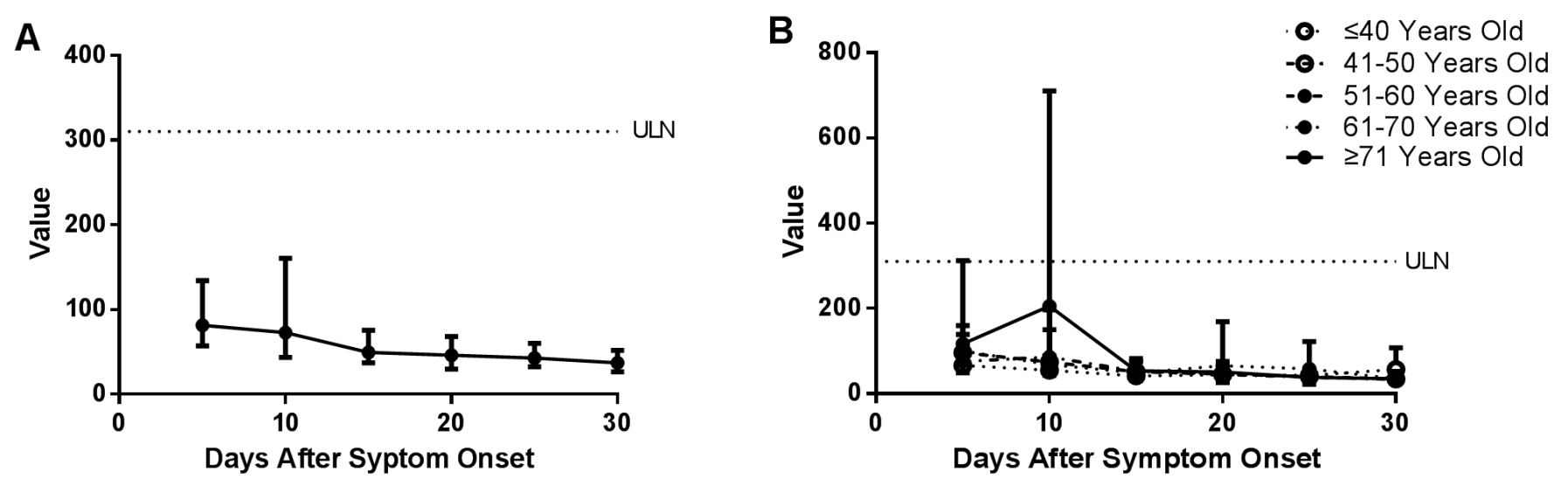

Supplement Figure The change trend of CK median value in 30 days (A) and in five age groups (B) 


\section{References:}

[1]. Huang, C., et al., Clinical features of patients infected with 2019 novel coronavirus in Wuhan, China. Lancet (London, England), 2020. $395(10223)$ : p. 497-506.

[2]. Zhu, N., et al., A Novel Coronavirus from Patients with Pneumonia in China, 2019. The New England journal of medicine, 2020. 382(8): p. 727-733.

[3]. Holshue, M.L., et al., First Case of 2019 Novel Coronavirus in the United States. The New England journal of medicine, 2020. $382(10)$ : p. 929-936.

[4]. McLaughlin, A.C., The interaction of 8-anilino-1-naphthalenesulfonate with creatine kinase. Evidence for cooperativitiy of nucleotide binding. The Journal of biological chemistry, 1974. 249(5): p. 1445-52.

[5]. Jacobus, W.E. and A.L. Lehninger, Creatine kinase of rat heart mitochondria. Coupling of creatine phosphorylation to electron transport. The Journal of biological chemistry, 1973. 248(13): p. 4803-10.

[6]. Wallimann, T., et al., Intracellular compartmentation, structure and function of creatine kinase isoenzymes in tissues with high and fluctuating energy demands: the 'phosphocreatine circuit' for cellular energy homeostasis. The Biochemical journal, 1992. 281(1): p. 21-40.

[7]. Perkoff, G.T., Demonstration of creating phosphokinase in human lung tissue. Archives of internal medicine, 1968. 122(4): p. 326-8.

[8]. Chen, N., et al., Epidemiological and clinical characteristics of 99 cases of 2019 novel coronavirus pneumonia in Wuhan, China: a descriptive study. Lancet (London, England), 2020. 395(10223): p. 507-513.

[9]. Li, X., et al., Cardiac injury associated with severe disease or ICU admission and death in hospitalized patients with COVID-19: a meta-analysis and systematic review. Critical care (London, England), 2020. 24(1): p. 468.

[10]. Wu, T., et al., Multi-organ Dysfunction in Patients with COVID-19: A Systematic Review and Meta-analysis. Aging and disease, $2020.11(4)$ : p. 874-894.

[11]. Li, M., et al., Cardiovascular disease potentially contributes to the progression and poor prognosis of COVID-19. Nutrition, metabolism, and cardiovascular diseases : NMCD, 2020. 30(7): p. 1061-1067. 
[12]. Zheng, F., et al., Clinical characteristics of 161 cases of corona virus disease 2019 (COVID-19) in Changsha. European review for medical and pharmacological sciences, 2020. 24(6): p. 3404-3410.

[13]. Chen, T., et al., Clinical characteristics of 113 deceased patients with coronavirus disease 2019: retrospective study. BMJ (Clinical research ed.), 2020. 368(undefined): p. m1091.

[14]. Zhou, F., et al., Clinical course and risk factors for mortality of adult inpatients with COVID-19 in Wuhan, China: a retrospective cohort study. Lancet (London, England), 2020. 395(10229): p. 1054-1062.

[15]. General Office Of Health Commission Of The People'S Republic Of China, O.O.N.A., Diagnosis and Treatment Protocol for Novel Coronavirus Pneumonia (Trial Version 7). Chinese medical journal, 2020. 133(9): p. 1087-1095.

[16]. Mahrholdt, H., et al., Presentation, patterns of myocardial damage, and clinical course of viral myocarditis. Circulation, 2006. 114(15): p. 1581-90. 